\title{
The Impact of Prostate Specific Antigen Testing on Incidence of Prostate Cancer cases in Zimbabwe
}

\author{
Article by Assam Musonza ${ }^{1}$, Professor Lynn Zijenah ${ }^{2}$, Victor T. Nyanhete ${ }^{3}$ \\ ${ }^{1} \mathrm{Ph} . \mathrm{D}$, in Clinical Research, Zimbabwe \\ ${ }^{2}$ P.O Box A178 Avondale Harare, Zimbabwe \\ ${ }^{3} 158$ Marks Road Daylesford Gweru, Midlands, Zimbabwe. \\ E-mail: assammuso@gmail.com / assammuso@yahoo.co.uk', lzijenah@gmail.com², \\ victort92@gmail.com ${ }^{3}$
}

\begin{abstract}
Prostate cancer is the leading cancer causing death in Zimbabwean men according to the Zimbabwe National Cancer Registry. By 2004 prostate cancer had become the most common cancer in Zimbabwean men. The incidence of prostate cancer shows strong age, race and geographical dependence. The prostate specific antigen (PSA) is a serine protease produced by cells of the prostate gland. The PSA blood test measures the level of PSA in man's blood and is used as a biomarker for prostate cancer (PCa). Unfortunately the PSA is prostate specific but not PCa specific. PSA testing started in Zimbabwe in 1995. The specific objectives of the study were: (i) to determine the age range most affected by PCa, (ii) to evaluate the impact of PSA testing on PCa cases in Zimbabwe. The study was a retrospective cross sectional study. Secondary data of all histology confirmed cases of PCa were obtained from the Zimbabwe National Cancer Registry (ZNCR) and analysed. Graph Pad Prism 7.03 was used to statistically analyse the data. The two-tailed T-test was employed to compare the number of pre-PSA cases against the number of post PSA era cases. 5277 PCa cases covering a thirty year period were retrieved. The first ten years (1986-1995) were the pre PSA era period and the next twenty years (1996-2015) were the post PSA era. The median age was 71 (IQR 64-78) years. There was a significant difference between number of PCa cases of the pre-PSA era (1986-1995) and the first post PSA era 1996-2005, and the second PSA era (2006-2015) ( $p=0.0042$ and $p=0.0028$ respectively. This showed the impact that PSA testing had on PCa in Zimbabwe (2006-2015). The study showed that the age group most affected by PCa was the 64-78 years. .
\end{abstract}

Keywords: Prostate cancer, Cross Sectional Study, Prostate Specific Antigen, PSA impact, age group, Zimbabwe.

\section{Introduction}

PCa also known as carcinoma of the prostate is the most common cancer among men only second to skin cancer in the world (Ferlay et al 2013). In Zimbabwe PCa is the leading cancer causing death in men (ZNCR report 2014)

\section{Risk factors}

The incidence of PCa shows strong age, race and geographical dependence (Center et al 2012 and Babb 2014. Autopsy studies have shown an age dependent risk of finding microscopic foci of what is considered to be PCa, from age 30 and up (Sakr, Haas, Cassin, Pontes and Crissman 1993), Soos, Tsakiris, Szanto, Turzo, Haas and Dezso 2005).

There is a large variation of the incidence of $\mathrm{PCa}$ in the world. The highest incidence is found in African-Americans and lowest in Chinese men (Hsing and Devesa 2001). The difference being about 40 fold.

The incidence rates of PCa in Africa are increasing at an alarming rate (Boyle and Levin 2008). PCa is the most commonly diagnosed cancer among men in Southern Africa and Western Africa including South Africa, Zimbabwe, Nigeria and Cameron (Jemal, Bray, Forman, O'Brien, Ferlay, Center and Parkin 2012). 
Heredity form of PCa constitutes approximately five per cent of the cases (Bratt, Kristofferson, Lundgren, and Olsson 1999). This is diagnosed on the pedigree with three relatives in different generations with the disease or three first degree relatives or early onset (before 55 years of age) among two relatives. The risk of dying from the disease increases if the relatives are young at diagnosis (Groomberg, Wiklund and Damber 1999). This raises the aspect of high incidence in AfricanAmericans and Africans in general, whether genetics plays a major role. It has not been established that this is the case but certain genes and DNA mutations have been implicated. A lot of work is underway to elucidate this. It has been established that there is no size fit all with regard to PCa treatment (Isaacs and Kainu, 2001).

In Zimbabwe, since 2004 PCa cases have increased by 104 per cent (ZNCR 2012 Report). An in depth understanding of which age group is most affected and what the impact of PSA testing on PCa cases is required. The study was undertaken to seek answers to these questions

\section{Screening for PCa}

The tests most commonly used to screen for PCa are the Digital Rectal Examination (DGE) and the PSA blood test. In the DGE, a doctor or nurse inserts a lubricated gloved finger into the rectum to estimate the size of the prostate and feel for lumps. This is very subjective and not very accurate. There are high chances of missing the tiny lumps and of under or overestimating the size of the prostate. Others use ultra sound scan to screen, the transrectal ultra sound scan is adjudged to be more superior. The PSA measures the level of PSA in the blood. The PSA is a serine protease normally secreted by the prostate in large amounts into the seminal plasma but only tiny amounts enter the blood stream hence low biological reference range of $0-4.0 \mathrm{ng} / \mathrm{ml}$. Prostatic diseases like PCa, Benign Prostatic Hypertrophy (BPH), urinary tract infections and prostatitis disrupt the integrity of the basal cell layer and basement membrane which leads to leakage of PSA into the blood stream (Andriole et al 2004). Therefore the PSA test is prostate specific but not PCa specific, which compromises its screening role. More biomarkers that are PCa specific and sensitive are required.

A biopsy is the main tool that's used to confirm and diagnose PCa. Small pieces of tissue are removed from the prostate and examined under the microscope by a histopathologist to check for cancer cells. A bone scan, computed tomography (CT or CAT) scan and Magnetic Resonance Imaging (MRI) are also used to find out if the cancer has spread.

The study used archived data collected and kept by the ZNCR. All confirmed PCa cases with their demographic data were retrieved electronically and the data was analysed.

\section{Methodology}

\section{Research design}

The study was a retrospective cross-sectional study using secondary data collected by the ZNCR. All confirmed PCa data obtained from the entire country was retrieved and analysed.

\section{Sampling technique}

A convenience type of sampling was employed. All the available data on prostate cancer from the ZNCR from 1986-2015 was retrieved and analysed.

\section{Data collection}

An electronic data set of all confirmed PCa patients was obtained from the ZNCR after the study was approved by the Medical Research Council of Zimbabwe (MRCZ). There were no names of patients to safeguard patient confidentiality. Information on each numbered patient included: registry number, year of diagnosis, age, race and district of residence and the histopathological diagnosis. The data set was from 1986 to 2015. 1986 -1995 was the pre PSA testing era and 1996-2015 was the post PSA testing era.

\section{Data analysis}

All the data was transferred onto an excel spread sheet and grouped into individual years (30) and the years in ascending order. The data was then grouped into five year groups for comparison using the 
two tailed T-test, the pre PSA years versus the post PSA era. The 1986 -1995 group was reference group for comparing with post PSA groups 1996-2005 and 2006-2015 groups. Graph Pad Prism 7.03 was employed to analyse the data. Significance level was $95 \%$ / p value 0.05 .

\section{Results}

A dataset with a total of 5277 confirmed PCa cases was obtained from the ZNCR on an excel spread sheet and analyses. There were 1191 (22.6\%) cases in the period January 1986 to December 1995. This was the era before PSA testing (pre-PSA era). 1996-2005 (first post-PSA era) had 37.0\% ( $\mathrm{n}=1955)$ PCa cases. And the second post-PSA era 2006-2015 had 40.4\% ( $\mathrm{n}=2131)$ cases (Fig 1 \& 2).

There was a significant difference in the number of cases between the pre-PSA era and the two postPSA eras ( $\mathrm{p}=0.0042$ and 0.0028 ) respectively. The number of cases between the two post-PSA periods, was not significantly different $(\mathrm{p}=0.8262)$.

The youngest patient was 9 years and the oldest was 99 years of age. The median age was 71 (IQR 64-78) years. There were $26(0.5 \%)$ PCa cases in the under 40 years age group, $116(2.2 \%)$ PCa cases in the 41-50 years age group, $703(13.3 \%)$ between 51-60 years of age and the over 60 years constituted $84 \%(\mathrm{n}=4432)$ of all the PCa cases (Fig 3).

Out of the 5277 confirmed cancer cases, 255 (4.83\%). had PSA results in their reports. Their median age was 76 (IQR 70-82) years. The median PSA level was 76.16 (IQR 32.95-287.29) ng/ml. 92\% of all the PCa cases were adenocarcinomas (Fig 4).

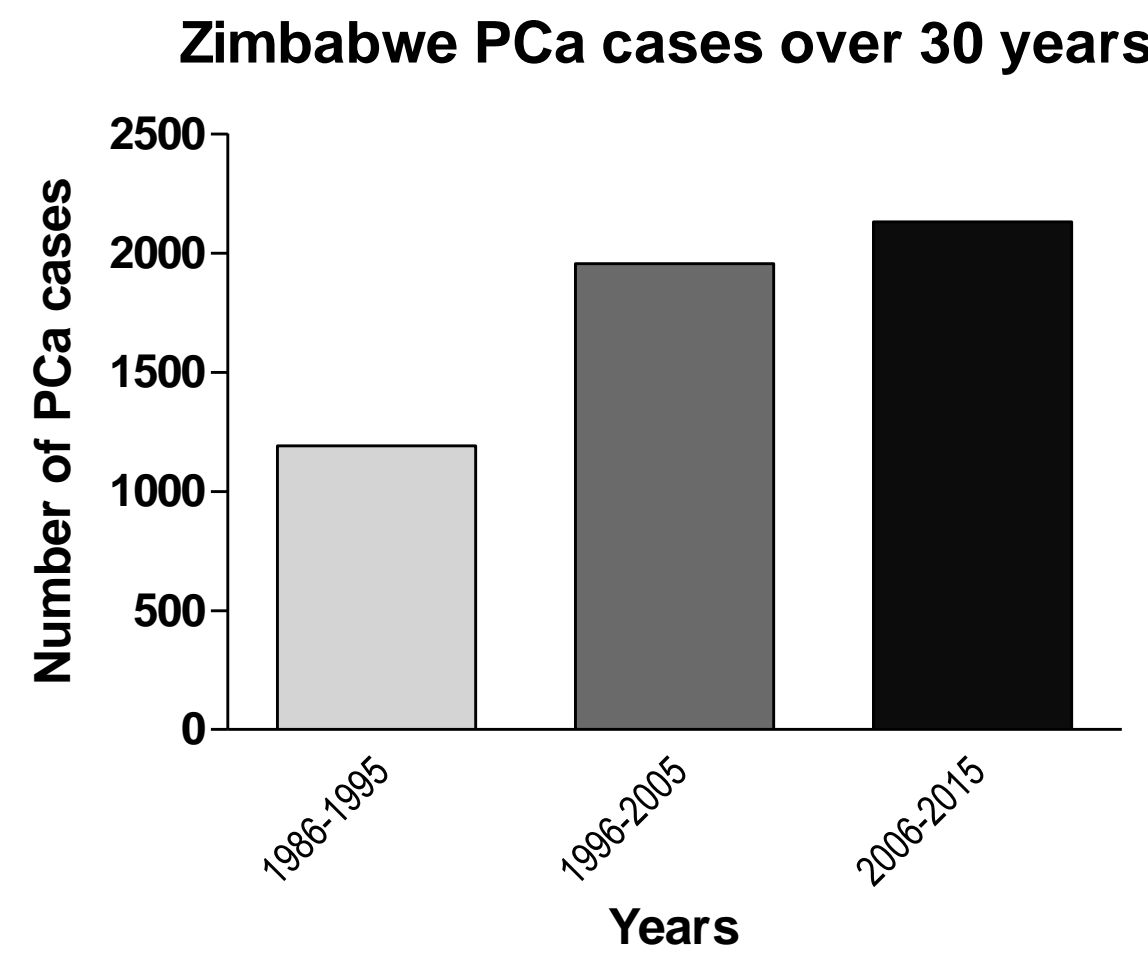

Figure 1: Total number of $\mathrm{PCa}$ cases over 30 years

$(\mathrm{n}=5277) 1986-1995(\mathrm{n}=1191) 1996-2005(\mathrm{n}=1955) 2006-2015(\mathrm{n}=2131)$ 
DOI: 10.21522/TIJCR.2014.04.02.Art006

ISSN: $2520-3096$ Prostate Cancer Cases in Z im bab we

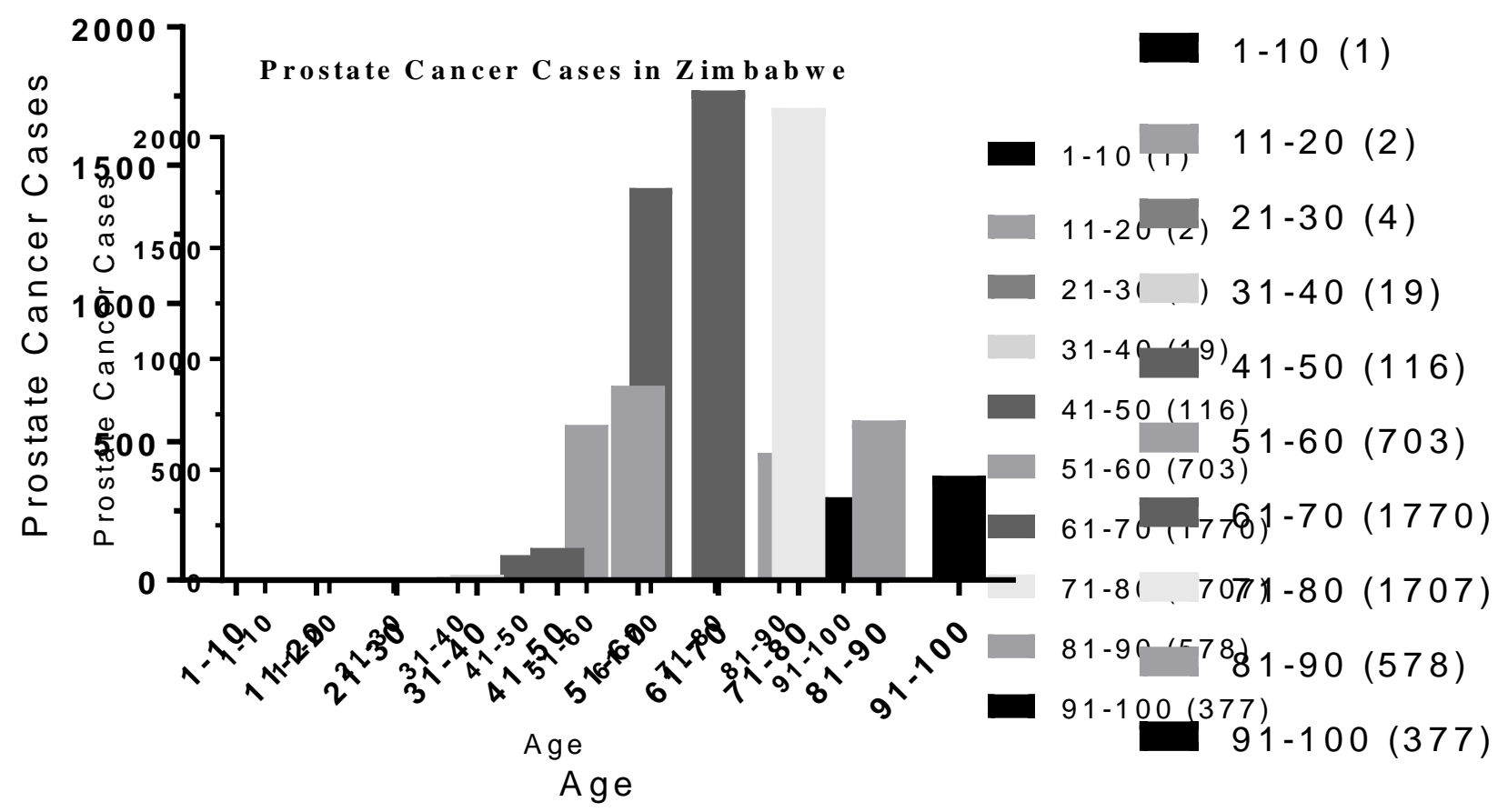

Figure 2. Prostate Cancer cases vs. Age in Zimbabwe. The number PCa cases per age group in brackets

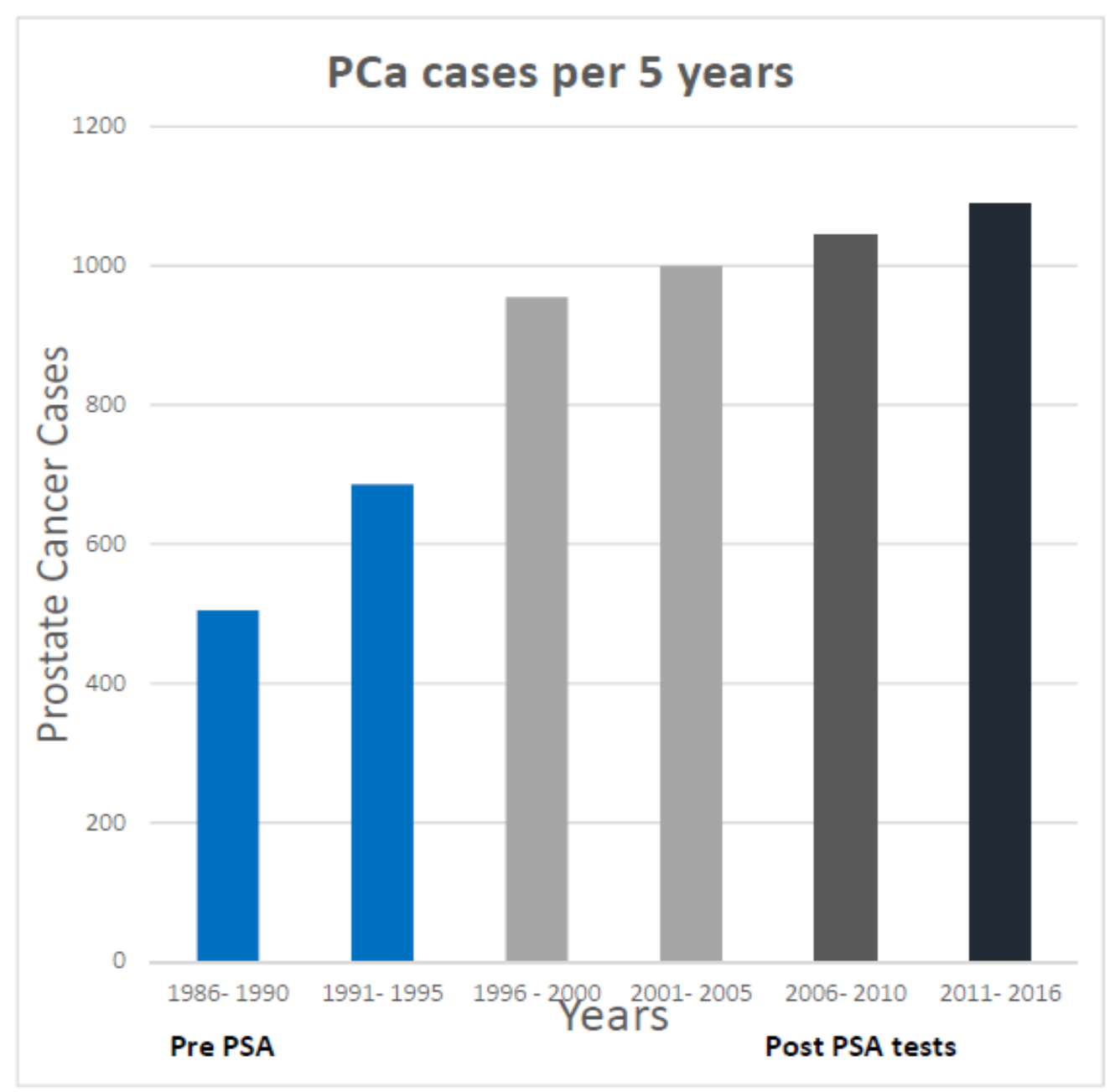

Figure 3. PCa cases per 5 years over 30 years 


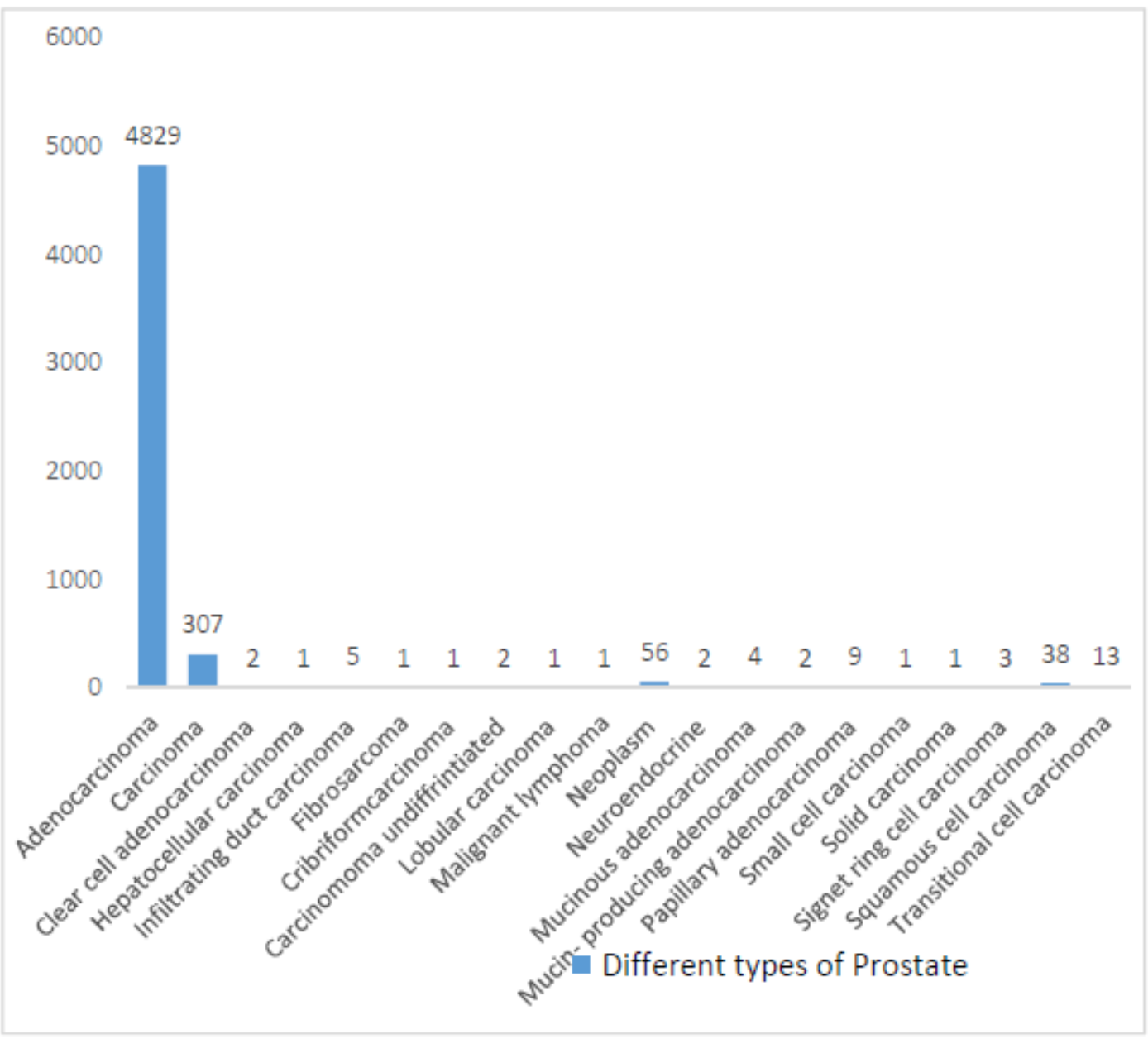

Figure 4. Types of PCa diagnosed over 30 years

92\% ( $\mathrm{n}=4829)$ Adenocarcinomas, 5.8\% ( $\mathrm{n}=38)$ squamous cell carcinoma.

\section{Discussion}

The study showed that the majority of the PCa cases were over 60 peaking at 99 years of age years of age confirming that PCa is a disease of the old in Zimbabwe just like it is in the rest of the world. The median age was 71 (IQR 64-78) years, meaning that fifty per cent of the PCa cases were in the 6478 years age group. According to these results PSA screening would really benefit the 40- 55 years age group considering the slow growing nature of most of the prostate tumours (Neal et al., 2000). Most PCAs found in comparatively young men (45-55 years) appear to be more aggressive and have high mortality rates (Salinas et al., 2014).

The $26(0.5 \%)$ PCa cases found in the under 40 years of age group would require further investigation as far as risk factors e.g., genetics are concerned. It has however been pointed out by Bubendorf et al ., (2000), and Soos et al ., (2005), that autopsy studies have shown an age dependant risk of finding microscopic foci of what is considered to be PCa from the age of 30 years. Considering their observation, the $0.5 \%$ PCa cases in the under 40 years would be expected.

The median age of 71 years is similar to the Swedish one of 69 (The National board of health and welfare Sweden 2008) and to the earlier study by this author (Musonza 2016) of 75 years (IQR 70-82) years using a much smaller sample size. In America the median age is 66 and in Europe it is 69 (Guzzo, Drach, Wein, 2016). These are all in agreement with results obtained in this study. There is no indication of ethnic or racial difference as far as the most affected age group is concerned.

PSA is organ specific and not PCa specific. Many men can live and die with PCa and not of PCa (Whitmore 1994). This is confirmed by the disparity for all ages between incidence and mortality rates e.g., in 199365000 men out of 165000 died in the USA (Whitmore 1994). The fact that most PCas are 
slow growing and indolent but will raise the PSA level which can lead to biopsies and treatment to an otherwise "friendly" tumour which would not cause any symptoms is a disadvantage of PSA testing. Other prostatic diseases like Benign Prostatic Hyperplasia (BPH), prostatitis and urinary tract infections can also raise the PSA levels (Caplan \& Kartz 2002).

The impact of PSA on the increase in PCa cases in Zimbabwe is demonstrated in the study. The study shows a significant increase in the number of PCa cases after the introduction of PSA testing from 1191 $(22.6 \%)$ cases to $1955(37 \%)$ and $2131(40.4 \%)$ p value $=0.0042$ and 0.0028 respectively. A comparison of the two ten year post PSA periods do not show any significant difference $37 \%$ compared to $40.4 \%$ ( $\mathrm{p}=0.8262$ ).

Therefore the introduction of PSA testing in 1996 had the effect of increasing the prevalence of PCa in Zimbabwe according to the results of this study. The non-specificity of PSA then brings in the debate of over diagnosis and over treatment. The United States Preventive Services Task Force (USPSTF) has issued guidelines discouraging screening using PSA (Moyer 2012). However PCa cases in the United States peaked after PSA testing was introduced, levelled off, new cases came down and mortalities declined (Moyer 2012). This debate about PSA screening requires more time and more studies in our own environment. There is need for more research on over diagnosis and over treatment because of PSA testing. At present early diagnosis using PSA screening has prolonged lives.

USPSTF and The Cancer Council of Australia have argued that the quality of life has been affected by procedures and over treatment that follow the treatment of indolent tumours as a result of PSA testing. Indolent tumours are slow growing and depending on the age, they would be outlived by other conditions with no symptoms at all. Watchful waiting has been proposed as a more practical way of dealing with raised PSA with no symptoms (Heijnsdijk, Wever and Auvinen et al 2002).

The introduction of PSA testing in Zimbabwe has revolutionised PCa diagnosis, treatment, monitoring treatment and prolongation of life. As more information is becoming available on the role of certain genes in PCa pathogenesis, more studies need to be carried out in Zimbabwe to combat men's number one cancer.

\section{Limitations and recommendations}

The use of secondary data, despite its cost effectiveness always has limitations because it would have been collected for other purposes like policy making, planning, and statistics or for answering different research questions, not the study's objectives. It is very difficult to exclude errors and omissions in the data if there are any. Certain vital information like treatments and outcomes was not available in this particular data set. It would have been very useful in the evaluation of the impact of age and PSA testing. The race and ethnic criteria was not taken into account due to the dominance by one major group.

It is recommended that more studies in collaboration with other groups be done to get more insight into PCa in Zimbabwe. The debate about over diagnosis and over treatment needs to be seriously looked at by all stakeholders including the patients themselves.

\section{Conclusion}

The age group most affected by PCa in Zimbabwe is the 64-78 years with a median age of 71 (IQR 64-78) years. $84 \%$ of all the PCa cases in this study were above 60 years of age. The introduction of PSA testing had the effect of suddenly increasing the number of PCa cases in Zimbabwe. There was a significant difference between the pre-PSA era and the two post-PSA eras $(\mathrm{p}=0.0042$ and $\mathrm{p}=0.0028)$ respectively. More studies are required on $\mathrm{PCa}$ for us to understand if there are other risk factors involved, now that we know the most affected age group. PCa is a major health problem in Zimbabwean men and needs better screening methods that detect the aggressive types of PCa, more awareness among men and access to diagnosis and treatment. 


\section{References}

[1].Andriole, G., Bostwick, D., Brawley, O., Gomella, L., Marberger, M. Tindall, D ..... (2004) Chemoprevention of Prostate Cancer in men at high risk: rational and design of the reduction by dutasteride of prostate cancer events (REDUCE) trial. J Urol: 172 (4PT1): 1314 - 7

[2].American Cancer Society (2009-2010). Cancer facts and figures for African-Americans (2009-2010). American Cancer Society. Atlanta.GA (2009).

[3].Babb, C., Urban, M., Kielkowsk, D., and Kellett, P. (2014). Prostate Cancer in South Africa: Pathology Based National Cancer Registry data (1986-2006) and Mortality rates (1997-2009). Prostate Cancer. Volume 2014, Article ID 419801.

[4].Boyle, P., \& Levin, B. (2008). World Cancer Report 2008. Lyon France: World Health Organisation, International Agency for Research on Cancer.

[5].Bratt, O., Kristofferson, U., Lundgren, R., Olsson, H., (1999). Familial and hereditary prostate cancer in southern Sweden. A population based case study. Eur J Cancer: 35 (2); 272 - 7.

[6].Bubendorf, L., Schöpfer, A., Wagner, U., Sauter, G., Moch, H., Willi, N., .. Mihatsch, M. J. (2000). Metastatic patterns of prostate cancer: An autopsy study of 1,589 patients. Human Pathology, 31(5), 578-583. https://doi.org/10.1053/hp.2000.6698.

[7].Caplan, A., Kartz. A. (2002). Prostate - specific antigen and the early diagnosis of Prostate Cancer. American Journal of Clinical Pathology. 117 (Supl: S104- 108).

[8].Center, M. M., Jemal, A., Lortet-Tieulent, J., Ward, E., Ferlay, J., Brawley, O et al (2012). International variation in prostate cancer incidence and mortality rates. Eur Urol, 61. 1079-1092.

[9].Guzzo, T.J Drach, G. W, Wein, A. J., (2016) Primer of Geriatric Urology.

[10]. Groomberg, H., Wiklund, F., Damber, J. E., (1999). Age specific risks of familial prostate carcinoma; a basis for screening recommendation in high risk populations. Cancer. 86 (3): $477-83$.

[11]. Heijnsdijk, E.A.M., Wever, E.M., Auvinen, A., Hugosson, J., Ciatto, S., Nelen, V., 2012). Quality - of Life Effects of Prostate- Specific- Antigen Screening. N Engl J Med; 367: 595 - 605.

[12]. Hsing, A.W., Devesa, S.S., (2001). Trends and Patterns of Prostate Cancer: What do they suggest? Epidemiol Rev; 23 (1): 3- 13.

[13]. Isaacs, W., \& Kainu, T. (2001). Oncogenes and tumour suppressor genes in prostate cancer. Epidemiol. Rev, Volume 23, pp 36-41.

[14]. Jemal, A., Bray, F., Forman, D., O’Brien, M., Ferlay, J., Center, M., \& Parkin, M. (2012). Cancer Burden in Africa and Opportunities for Prevention. Cancer. DOI; 10. 1002/cncr. 27410, Received August 10 2011; Revised: October 24, 2011, published online in Wiley Online Library (wileyonlinelibrary.com).

[15]. Moyer, V, A., (2012). US. Preventive Services Task Force. Screening for prostate cancer: US. Preventive Services Task Force recommendation statement. Ann Intern Med. 157; 120-34.

[16]. Neal, D. ., Neal, C. information about the author D. ., \& Neal, Email the author D.E, H.Y Leung, P.H Powell, F.C Hamdy, J. D. (2000). Unanswered questions in screening for prostate cancer Neal 2000 - European Journal of Cancer. https://doi.org/http://dx.doi.org/10.1016/S0959-8049 (00)00104-0.

[17]. Sakr, W. A., Haas, G. P., Cassin, B.F., Pontes, J. E., Crissman, J.D. (1993). The frequency of carcinoma and intraepithelial neoplasia of the prostrate in young patients. J. Urol: 150(2) Pt 1: 379- 385.

[18]. Salinas, C. A., Tsodikov, A., Ishak-Howard, M., \& Cooney, K. A. (2014). Prostate cancer in young men: an important clinical entity. Nature Reviews. Urology. https://doi.org/10.1038/nrurol.2014.91.

[19]. Soos, G., Taskiris, I., Szanto, J., Turzo, C., Haas, P. G., and Dezso, B. (2005). The prevalence of prostate carcinoma and its precursor in Hungary; an autopsy study. Eur Urol: 48 (5): 739 - 44.

[20]. The National board of health and welfare. Cancer Prevalence Riket. (2008).

[21]. Whitmore, W. F. (1994). Localised prostatic cancer: Management and detection issues. Lancet. https://doi.org/10.1016/S0140-6736(94)92156-3.

[22]. Zimbabwe National Cancer Registry Report (2014). 